\title{
A DISCRETE MODEL OF EQUIVARIANT STABLE HOMOTOPY FOR CYCLIC GROUPS
}

\author{
CHRISTIAN SCHLICHTKRULL
}

\begin{abstract}
Given a space $X$, the Barratt-Eccles construction $\Gamma(X)$ provides a simplicial model for the infinite loop space colim $\Omega^{n}\left(\Sigma^{n} X\right)$ representing stable homotopy of $X$. In this paper we extend this construction to give an endofunctor on the category of spaces with a cyclic structure in the sense of A. Connes. More generally we consider spaces $X$ with an action of a finite cyclic group $\mathrm{C}_{r}$, and we show how to impose on $\Gamma(X)$ a natural $r$-action. This gives a model for the equivariant infinite loop space colim $\Omega^{V}\left(\Sigma^{V} X\right)$, where $V$ runs through the finite dimensional representations of $\mathrm{C}_{r}$. In particular we get a useful discrete model of the equivariant suspension spectrum of a cyclic set.
\end{abstract}

\section{Contents}

0. Introduction

1. Preliminaries on $\Gamma^{+}(X)$ and $\Gamma(X)$

2. The equivariant structure

3. The Segal-tom Dieck splitting

4. Cofibration sequences

5. The R-map

6. The Wirthmüller Isomorphism

7. Comparison with equivariant infinite loop spaces

\section{Introduction}

The $\Gamma$-construction was introduced by Barratt and Eccles in [BE], where they proved that $\Gamma(X)$ is a simplicial model for $\mathrm{Q}(X)=\lim \Omega^{n} \Sigma^{n}(X)$ for any pointed space $X$. In this paper we shall generalize this to spaces with a basepoint-preserving action of a finite cyclic group $\mathrm{C}_{r}$. For such $X$ we have the following definition of the equivariant infinite loop space:

$$
\mathrm{Q}_{\mathrm{C}_{\mathrm{r}}}(X)=\lim _{\rightarrow} \operatorname{Map}\left(S^{l \mathscr{R}}, S^{l \mathscr{R}} \wedge X\right),
$$

where $\mathscr{R}=\mathrm{R}\left[\mathrm{C}_{r}\right]$ is the real regular representation of $\mathrm{C}_{r}$ and $S^{l \mathscr{R}}$ is the one- 
point compactification of $l \mathscr{R}=\bigoplus_{\nu=1}^{l} \mathscr{R}$. With this notation we can state our main theorem.

THeorem 7.1. For a pointed $\mathrm{C}_{r}$-space $X$ there is a natural $\mathrm{C}_{r}$-action on $|\Gamma(X)|$, the topological realization of $\Gamma(X)$, such that $|\Gamma(X)|$ and $\mathrm{Q}_{\mathrm{C}_{r}}(X)$ are equivariantly homotopy equivalent.

The $\mathrm{C}_{r}$-action on $|\Gamma(X)|$ comes about by combining the $\mathrm{C}_{r}$-action on $X$ with a certain cyclic structure on the $\Gamma$-construction. Recall that for a space $Z$ with a cyclic structure in the sense of A. Connes, the realization $|Z|$ has a natural $S^{1}$-action. We shall prove the following result.

COROLlary 7.2. For a pointed cyclic space $Z$ there is an induced cyclic structure on $\Gamma(Z)$. The realizations $|Z|$ and $|\Gamma(Z)|$ thus have natural $S^{1}$-actions, and for any finite subgroup $\mathrm{C}_{r}$, the $\mathrm{C}_{r}$-spaces $|\Gamma(Z)|$ and $\mathrm{Q}_{\mathrm{C}_{r}}(|Z|)$ are equivariantly homotopy equivalent.

However, $\Gamma(Z)$ is definitively not a model for $\mathrm{Q}_{S^{1}}(|Z|)$ since the $S^{1}$-fixedpoints of $|\Gamma(Z)|$ is a discrete subset of the vertices in the cyclic space $\Gamma(Z)$, and therefore different from the fixed-points of $\mathrm{Q}_{S^{1}}(|Z|)$, cf. [tD].

From the viewpoint of $\mathrm{C}_{r}$-equivariant stable homotopy the $\Gamma$-construction is a combinatorial substitute for $\mathrm{Q}_{\mathrm{C}_{r}}(X)$. In particular, we get by using the $\Gamma$-construction a combinatorial version of the Segal-tom Dieck splitting:

$$
\mathrm{Q}_{\mathrm{C}_{r}}(X)^{\mathrm{C}_{r}} \cong \prod_{t \mid r} \mathrm{Q}\left(\mathrm{EC}_{r / t_{+}} \wedge_{\mathrm{C}_{r / t}} X^{\mathrm{C}_{t}}\right)
$$

cf. [BHM, 5.17]. Namely, the edgewise subdivision functor $\mathrm{sd}_{r}$ (to be recalled in Section 2) turns $\Gamma(X)$ into a simplicial space with a simplicial $\mathrm{C}_{r}$-action, and we construct in Section 3 an explicit homotopy equivalence

$$
\prod_{t \mid r} \Gamma\left(\mathrm{EC}_{r / t_{+}} \wedge_{\mathrm{C}_{r / t}} X^{\mathrm{C}_{t}}\right) \rightarrow \mathrm{sd}_{r} \Gamma(X)^{\mathrm{C}_{r}} .
$$

In the special case where $X$ is a pointed free $\mathrm{C}_{r}$-space, the equivalence $\mathrm{Q}\left(X / \mathrm{C}_{r}\right) \simeq \mathrm{Q}_{\mathrm{C}_{r}}(X)^{\mathrm{C}_{r}}$ (cf. [A, 5.4]) is realized by a homomorphism of simplicial free groups:

$$
\Gamma\left(X / \mathrm{C}_{r}\right) \cong \Gamma\left(\mathrm{EC}_{r+} \wedge_{\mathrm{C}_{r}} X\right) \rightarrow \operatorname{sd}_{r} \Gamma(X)^{\mathrm{C}_{r}} .
$$

On our way to proving Theorem 7.1 we shall in fact cover the basic part of $\mathrm{C}_{r}$-equivariant stable homotopy theory in the framework of the $\Gamma$-construction. After constructing the Segal-tom Dieck splitting in Section 3 we show in Section 4 that $\Gamma$ turns equivariant cofibration sequences into homotopy fibrations. In Section 5 we define the restriction map 


$$
\mathrm{R}: \mathrm{sd}_{r} \Gamma(X)^{\mathrm{C}_{r}} \rightarrow \mathrm{sd}_{r / s} \Gamma\left(X^{\mathrm{C}_{s}}\right)^{\mathrm{C}_{r / s}}
$$

for a subgroup in $\mathrm{C}_{r}$. This is the simplicial analogue of the map

$$
\text { fix : } \mathrm{Q}_{\mathrm{C}_{r}}(X)^{\mathrm{C}_{r}} \rightarrow \mathrm{Q}_{\mathrm{C}_{r / s}}\left(X^{\mathrm{C}_{s}}\right)^{\mathrm{C}_{r / s}}, \quad \operatorname{fix}(f)=f^{\mathrm{C}_{s}},
$$

which restricts a map to the $\mathrm{C}_{s}$ fixed-points. This map is fundamental in the definition of the topological cyclic homology functor TC due to M. Bökstedt, W.C. Hsiang and I. Madsen [BHM]. We shall see in [Sch1] and [Sch2] how the $\Gamma$-construction (or rather the $\Gamma^{+}$-construction, cf. Section 1) can be used to construct a new model $\mathrm{TC}^{+}$with good properties, including an explicit trace map inducing Morita equivalence. In section 6 we prove the Wirthmüller isomorphism for $\Gamma$, and finally in Section 7, we return to the comparison with $\mathrm{Q}_{\mathrm{C}_{r}}(X)$ and prove Theorem 7.1 above.

Spaliński [Sp] has given the category of cyclic sets a closed model category structure such that the weak equivalences are the maps that induce an equivalence on fixed-points for all finite subgroups of $S^{1}$. In this paper the interest is in explicit space-level constructions, and we do not use the abstract language of model categories.

The equivariant structure of $\Gamma(X)$ was hinted at in the above mentioned paper [BHM]. I want to thank I. Madsen for tutorials on equivariant homotopy theory.

\section{Preliminaries on $\Gamma^{+}(X)$ and $\Gamma(X)$}

We shall work in the category of pointed simplicial sets, but for convenience we freely adapt topological language such as spaces, subspaces etc. when talking about simplicial objects. The results of this paper are then transported into the category of equivariant $\mathrm{CW}$-complexes by means of the pair of adjoint functors, topological realization $|\cdot|$ and singular complex sin.. In fact we could also work directly with topological spaces, at least when applying the functor $\Gamma^{+}$defined below, which is sufficient to handle the case of connected spaces.

For the convenience of the reader we begin by recalling some elementary combinatorial facts. Let $\mathscr{M}$ be the category with objects the finite sets $\mathbf{n}=\{1, \ldots, n\}$ and morphisms $\mathscr{M}(\mathbf{m}, \mathbf{n})$ the strictly increasing maps from $\mathbf{m}$ to $\mathbf{n}$. Sometimes it will convenient to denote an object in $\mathscr{M}$ as $(n]=\{1, \ldots, n\}$ instead of using bold-face letters; for example we prefer to write $(m n]$ instead of $\mathbf{m n}$. This notation should be compared with the usual notation in the simplicial category, where the objects are usually denoted by $[n]=\{0, \ldots, n\}$.

The group of permutations of $\mathbf{n}$ is denoted by $\Sigma_{n}$. For $\sigma \in \Sigma_{n}$ and 
$\alpha \in \mathscr{M}(\mathbf{m}, \mathbf{n})$ the composite $\sigma \alpha$ is not necessarily strictly increasing, but there is a unique morphism $\sigma_{*}(\alpha) \in \mathscr{M}(\mathbf{m}, \mathbf{n})$ with $\sigma_{*}(\alpha)(\mathbf{m})=\sigma \alpha(\mathbf{m}) \subseteq \mathbf{n}$.

Definition 1.1. For $\alpha \in \mathscr{M}(\mathbf{m}, \mathbf{n})$ we define the restriction map $\alpha^{*}: \Sigma_{n} \rightarrow \Sigma_{m}$ by commutativity of the diagram



The action of $\alpha^{*}$ can also be seen more intuitively as follows: Write the numbers $1, \ldots, n$ in their natural order in two columns, one beside the other, and represent $\sigma$ by a set of arrows pointing from $s$ to $\sigma(s)$. Then delete all those arrows which has domain not in the image of $\alpha$, and delete also their domain and codomain. We keep the remaining arrows in their natural order, and by renumbering we get the desired element in $\Sigma_{m}$.

The restriction map $\alpha^{*}$ is not a group homomorphism, but it satisfies the following condition.

$$
\alpha^{*}(\tau \sigma)=\left(\sigma_{*} \alpha\right)^{*}(\tau) \circ \alpha^{*}(\sigma) \quad \text { for } \tau, \sigma \in \Sigma_{n} \text { and } \alpha \in \mathscr{M}(\mathbf{m}, \mathbf{n}) .
$$

Next we consider the cycle decomposition of a permutation $\sigma \in \Sigma_{n}$. The cyclic subgroup of $\Sigma_{n}$ generated by $\sigma$ acts on $\mathbf{n}$, and gives a decomposition of $\mathbf{n}$ into orbits $\mathbf{n}=J_{1} \amalg \ldots \amalg J_{k}$. The cycle decomposition $\sigma=\sigma_{1} \circ \ldots \circ \sigma_{k}$ is induced from this with $\sigma_{i}$ acting on $J_{i}$ by cyclic permutation. With this notation suppose we have $\alpha \in \mathscr{M}(\mathbf{m}, \mathbf{n})$ such that $\alpha(\mathbf{m})=J_{i_{1}} \amalg \ldots \amalg J_{i_{\nu}}$. Then it is easy to see that

$$
\sigma_{*}(\alpha)=\alpha,
$$

and that $\alpha^{*}(\sigma)$ has cycle decomposition

$$
\alpha^{*}(\sigma)=\alpha^{*}\left(\sigma_{i_{1}}\right) \circ \ldots \circ \alpha^{*}\left(\sigma_{i_{\nu}}\right) .
$$

Notice that 1.3 implies that $\alpha^{*}(\tau \sigma)=\alpha^{*}(\tau) \circ \alpha^{*}(\sigma)$ for any $\tau \in \Sigma_{n}$.

Definition 1.5. For a based simplicial set $X$ there is a right action of $\Sigma_{n}$ on $X^{n}$ given by

$$
\left(x_{1}, \ldots, x_{n}\right) \sigma=\left(x_{\sigma(1)}, \ldots, x_{\sigma(n)}\right)
$$

for $\sigma \in \Sigma_{n}$. Similarly, a morphism $\alpha \in \mathscr{M}(\mathbf{m}, \mathbf{n})$ induces a map $\alpha^{*}: X^{n} \rightarrow X^{m}$ by letting

$$
\alpha^{*}\left(x_{1}, \ldots, x_{n}\right)=\left(x_{\alpha(1)}, \ldots, x_{\alpha(m)}\right) .
$$


Given $\mathbf{x} \in X^{n}$ we say that $\alpha$ is entire for $\mathbf{x}$ if $\alpha^{*}$ only misses the basepoint, i.e. $i \notin \alpha(\mathbf{m})$ implies $x_{i}=*$.

Definition 1.6. Let $\mathrm{E}$ be the functor from sets to cyclic sets given by

$$
\mathrm{E} X:[i] \mapsto \operatorname{Map}([i], X)=X^{i+1}
$$

for any set $X$. The cyclic structure maps are given as follows:

$$
\begin{aligned}
d_{\nu}\left(x_{0}, \ldots, x_{i}\right) & =\left(x_{0}, \ldots, \hat{x}_{\nu}, \ldots, x_{i}\right) \\
s_{\nu}\left(x_{0}, \ldots, x_{i}\right) & =\left(x_{0}, \ldots, x_{\nu}, x_{\nu}, \ldots, x_{i}\right) \\
t_{i}\left(x_{0}, \ldots, x_{i}\right) & =\left(x_{i}, x_{0}, \ldots, x_{i-1}\right) .
\end{aligned}
$$

(We refer the reader to [L] for the general theory of cyclic sets.) As a simplicial set $\mathrm{E} X$ is contractible for all $X$. In the special case where $X$ is a discrete group $G$ there is a free right action of $G$ on $\mathrm{E} G$, and this makes the realization a model for the universal $G$ bundle. Notice also, that the restriction map 1.1 extends to a cyclic map $\alpha^{*}: \mathrm{E} \Sigma_{n} \rightarrow \mathrm{E} \Sigma_{m}$, using the functoriality of $\mathrm{E}$.

For $X$ a pointed simplicial set we have the bisimplicial set

$$
\mathscr{U}(X)=\coprod_{n \geq 0} \mathrm{E} \Sigma_{n} \times X^{n},
$$

where $X^{n}$ denotes the simplicial diagonal in the multisimplicial set $X^{n}$. Consider the following relations on $\mathscr{U}(X)$ :

$$
\begin{array}{lll}
\text { (i) } & (e, \mathbf{x}) \sim(e \sigma, \mathbf{x} \sigma) & \text { for } e \in \mathrm{E} \Sigma_{n}, \mathbf{x} \in X^{n} \text { and } \sigma \in \Sigma_{n} \\
\text { (ii) } \quad(e, \mathbf{x}) \sim\left(\alpha^{*} e, \alpha^{*} \mathbf{x}\right) & & \text { for } e \in \mathrm{E} \Sigma_{n}, \mathbf{x} \in X^{n} \text { and } \alpha \in \mathscr{M}(\mathbf{m}, \mathbf{n}) \\
& & \text { entire for } \mathbf{x} .
\end{array}
$$

Definition 1.8. [BE]. The bisimplicial set $\Gamma^{+}(X)$ has $(i, j)$ simplices

$$
\Gamma_{i}^{+}\left(X_{j}\right)=\coprod_{n \geq 0} \mathrm{E}_{i} \Sigma_{n} \times X_{j}^{n} / \sim
$$

where $\sim$ is the equivalence relation generated by (i) and (ii).

The elements in $\Gamma^{+}(X)$ are denoted $[e ; \mathbf{x}]$ for $e \in \mathrm{E} \Sigma_{n}$ and $x \in X^{n}$. In the following we shall often consider $\Gamma^{+}(X)$ as a simplicial set by restricting to the simplicial diagonal.

We next recall some general facts about group completion. The group completion $\mathrm{U}(M)$ of a monoid $M$ is the free group generated by the elements in $M$ modulo the relations $\langle x y\rangle \sim\langle x\rangle\langle y\rangle$ for $x, y \in M$. Clearly $\mathrm{U}$ gives a functor from monoids to groups, and $M \rightarrow \mathrm{U}(M)$ is universal among 
monoid homomorphisms from $M$ to groups. When $M$ is a simplicial monoid $\mathrm{U}$ applies in each simplicial degree and gives a simplicial group $\mathrm{U}(M)$.

For any simplicial monoid $M$, the inclusion $\mathrm{Z}\left[\pi_{0}(M)\right] \rightarrow \mathrm{H}_{*}(M)$ makes $\mathrm{H}_{*}(M)$ a $\mathrm{Z}\left[\pi_{0}(M)\right]$ module. (We shall always use homology with integer coefficients.) Using the Pontrjagin ring structure of $\mathrm{H}_{*}(\mathrm{U}(M))$, we get a natural map

$$
\mathrm{H}_{*}(M) \otimes_{\mathrm{Z}\left[\pi_{0}(M)\right]} \mathrm{Z}\left[\pi_{0}(\mathrm{U}(M))\right] \rightarrow \mathrm{H}_{*}(\mathrm{U}(M)) .
$$

The following theorem is due to Quillen.

THeOREM 1.10 [BE, 5.2]. If (a) $M$ is a free simplicial monoid, and (b) $\pi_{0}(M)$ is in the centre of the ring $H_{*}(M)$, then 1.9 is a ring isomorphism.

The simplicial monoids we are going to consider will be homotopy commutative, and (b) in the theorem is then automatically satisfied. However, we will also have to consider products of free simplicial monoids, and since taking products does not preserve freeness we need the following result.

COROLlaRY 1.11. If $M$ is a finite product of homotopy-commutative free simplicial monoids then 1.9 is an isomorphism.

Proof. It suffices to consider the product $M$ of two homotopy commutative free simplicial monoids $M_{1}$ and $M_{2}$. In the proof we need the following observation.

(1.12) For a commutative (discrete) monoid $N$ the inclusion of rings $\mathrm{Z}[N] \rightarrow \mathrm{Z}[\mathrm{U}(N)]$ makes $\mathrm{Z}[\mathrm{U}(N)]$ a flat $\mathrm{Z}[N]$-module.

To see this we use the translation category $\mathscr{X}$ of $N$. It has objects the elements in $N$, and a morphism $c$ from $a$ to $b$ is an element $c \in N$ with $c a=b$. As an $N$-set we can identify $\mathrm{U}(N)$ with the direct limit of the functor from $\mathscr{X}$ that is constantly equal to $N$ on objects and acts by left multiplication on morphisms. It follows that $\mathrm{Z}[\mathrm{U}(N)]$ is a direct limit of free $\mathrm{Z}[N]$-modules and therefore flat.

We return to the proof of 1.11 and write $A_{i}=\mathrm{Z}\left[\pi_{0}\left(M_{i}\right)\right]$ and $\widetilde{A}_{i}=\mathrm{Z}\left[\pi_{0}\left(\mathrm{U}\left(M_{i}\right)\right)\right]$ for $i=1,2$. Clearly $A_{1} \otimes A_{2} \cong \mathrm{Z}\left[\pi_{0}(M)\right]$ and since $\mathrm{U}(M) \cong \mathrm{U}\left(M_{1}\right) \times \mathrm{U}\left(M_{2}\right)$ we also have $\widetilde{A}_{1} \otimes \widetilde{A}_{2} \cong \mathrm{Z}\left[\pi_{0}(\mathrm{U}(M))\right]$. From the above discussion it follows that $\widetilde{A}_{1} \otimes \widetilde{A}_{2}$ is a flat $A_{1} \otimes A_{2}$ module. The Künneth exact sequence for the product $M=M_{1} \times M_{2}$ is a sequence of $A_{1} \otimes A_{2}$ modules, and by tensoring with $\widetilde{A}_{1} \otimes \widetilde{A}_{2}$ we get the following exact sequence: 
A DISCRETE MODEL OF EQUIVARIANT STABLE...

$$
\begin{aligned}
0 \rightarrow \bigoplus_{i+j=n} \mathrm{H}_{i}\left(M_{1}\right) & \otimes_{A_{1}} \widetilde{A}_{1} \otimes \mathrm{H}_{j}\left(M_{2}\right) \otimes_{A_{2}} \widetilde{A}_{2} \rightarrow \mathrm{H}_{n}(M) \otimes_{A_{1} \otimes A_{2}} \widetilde{A}_{1} \otimes \widetilde{A}_{2} \\
& \rightarrow \bigoplus_{i+j=n-1} \operatorname{Tor}\left(\mathrm{H}_{i}\left(M_{1}\right) \otimes_{A_{1}} \widetilde{A}_{1}, \mathrm{H}_{j}\left(M_{2}\right) \otimes_{A_{2}} \widetilde{A}_{2}\right) \rightarrow 0
\end{aligned}
$$

The result now follows from 1.10 and the five-lemma by comparing with the Künneth exact sequence for $\mathrm{U}(M) \cong \mathrm{U}\left(M_{1}\right) \times \mathrm{U}\left(M_{2}\right)$.

COROLlary 1.13. Let $M_{1}$ and $M_{2}$ be finite products of homotopy-commutative free simplicial monoids, and let $f: M_{1} \rightarrow M_{2}$ be a homomorphism. Then if $f$ induces an isomorphism on homology so does $\mathrm{U}(f)$. In particular this implies that $\mathrm{U}(f)$ is a homotopy equivalence.

Proof. The first statement follows from the naturality of 1.9 and Corollary 1.11. The second statement follows from the first, since in general integral homology isomorphism implies homotopy equivalence for a homomorphism of simplicial groups.

Given $e \in \Sigma_{m}$ and $f \in \Sigma_{n}$, we let $e \oplus f \in \Sigma_{m+n}$ be the permutation that acts by $e$ on the first $m$ elements and by $f$ on the last $n$ elements. There is a natural product on $\Gamma^{+}(X)$ :

$$
\begin{aligned}
& \mu: \Gamma^{+}(X) \times \Gamma^{+}(X) \rightarrow \Gamma^{+}(X) \\
& \mu([e ; \mathbf{x}],[f ; \mathbf{y}])=[e \oplus f ; \mathbf{x}, \mathbf{y}],
\end{aligned}
$$

and by [BE, 3.9 and 3.11] this gives $\Gamma^{+}(X)$ the structure of a homotopycommutative free simplicial monoid.

Definition 1.15 [BE]. For a pointed simplicial set $X, \Gamma(X)=\mathrm{U} \Gamma^{+}(X)$.

For $Y$ a topological space let $\Sigma^{n} Y$ denote the smash product of $Y$ with the realization of the $n$-fold smash product of some simplicial model for $S^{1}$. M. Barratt and $\mathrm{P}$. Eccles constructs a weak equivalence between $|\Gamma(X)|$ and $\mathrm{Q}(|X|)=\lim _{\rightarrow} \Omega^{n}\left(\Sigma^{n}|X|\right)$ in the following two steps.

Theorem 1.16 [BE, 4.9]. The inclusion $\Sigma^{n} X \rightarrow \Gamma\left(\Sigma^{n} X\right)$ induces a homotopy equivalence

$$
\lim _{\rightarrow} \Omega^{n}\left(\Sigma^{n}|X|\right) \rightarrow \lim _{\rightarrow} \Omega^{n}\left(\left|\Gamma\left(\Sigma^{n} X\right)\right|\right) .
$$

TheOREm 1.17 [BE, 4.7]. The stabilization map $S^{n} \wedge \Gamma(X) \rightarrow \Gamma\left(\Sigma^{n} X\right)$ induces by adjunction a map $|\Gamma(X)| \rightarrow \Omega^{n}\left(\left|\Gamma\left(\Sigma^{n} X\right)\right|\right)$, which is a homotopy equivalence. In particular this gives a homotopy equivalence

$$
|\Gamma(X)| \rightarrow \lim _{\rightarrow} \Omega^{n}\left(\left|\Gamma\left(\Sigma^{n} X\right)\right|\right) .
$$




\section{The equivariant structure}

We begin with the notion of a cyclic set. This is a simplicial set $Z$ together with extra cyclic operators $t_{i}: Z_{i} \rightarrow Z_{i}$, compatible with the simplicial structure of $Z$, cf. [L, 6.1.2]. The important fact about cyclic sets is that their topological realization admits a natural $S^{1}$-action. In particular there is an action of every finite cyclic group $\mathrm{C}_{r}$, and it is these actions we shall be concerned with. We shall always consider the finite cyclic groups as subgroups of the circle group $S^{1}$. This gives a natural generator $\mathrm{T}=\exp (i 2 \pi / r)$ for $\mathrm{C}_{r}$, and therefore also a canonical isomorphism $\mathrm{C}_{r} / C_{s} \cong \mathrm{C}_{r / s}$ whenever $s$ divides $r$.

Lemma 2.1. Let $X$ be a $\mathrm{C}_{r}$-space. Then the realizations $\left|\Gamma^{+}(X)\right|$ and $|\Gamma(X)|$ have natural $S^{1} \times \mathrm{C}_{r}$-actions, and thus also a diagonal $r$-action.

Proof. Notice that $\Gamma^{+}(X)$ is a bisimplicial set, with one simplicial direction coming from $\mathrm{E} \Sigma_{n}$ and the other from $X$. For $j$ fixed $\Gamma^{+}\left(X_{j}\right)$ is thus a simplicial set with a cyclic structure inherited by the cyclic structure on $\mathrm{E} \Sigma_{n}$, cf. 1.6.

The $S^{1}$-action on the realization $\left|\Gamma^{+}\left(X_{j}\right)\right|$ extends by functoriality to a $S^{1} \times \mathrm{C}_{r}$-action with $\mathrm{C}_{r}$ acting on $X_{j}$. Thus $[j] \mapsto\left|\Gamma^{+}\left(X_{j}\right)\right|$ becomes a simplicial $S^{1} \times \mathrm{C}_{r}$-space. The desired action on $\left|\Gamma^{+}(X)\right|$ is the induced simplicial action, noting that the topological realization of a bisimplicial set may be formed in two steps:

$$
\left|\Gamma^{+}(X)\right|=|j \mapsto| \Gamma^{+}\left(X_{j}\right)||
$$

For $\Gamma=\mathrm{U} \Gamma^{+}$, the lemma follows by functoriality of $\mathrm{U}$.

Since we are only interested in the actions of the finite cyclic groups, it will be convenient to apply the edgewise-subdivision functor $\mathrm{sd}_{r}$ from [BHM, Section 1]. This functor associates to every cyclic space $Z$ a new space $\operatorname{sd}_{r}(Z)$ with a simplicial $\mathrm{C}_{r}$-action, together with a $\mathrm{C}_{r}$-equivariant homeomorphism

$$
\mathrm{D}:\left|\operatorname{sd}_{r}(Z)\right| \rightarrow|Z| \text {. }
$$

The advantage of this is that the somewhat complicated $\mathrm{C}_{r}$-action on $|Z|$ is reduced to a simplicial action on $\operatorname{sd}_{r}(Z)$. In particular we have for the cyclic set $\mathrm{E} \Sigma_{n}$ that $\operatorname{sd}_{r} \mathrm{E} \Sigma_{n}=\mathrm{E} \Sigma_{n}^{r}$, with $\mathrm{C}_{r}$-action induced from the cyclic permutation of the $r$ factors.

Definition 2.3. The functor $\operatorname{sd}_{r} \Gamma^{+}$associates to a simplicial set $X$ the bisimplicial set

$$
\operatorname{sd}_{r} \Gamma^{+}(X)=\coprod_{n \geq 0} \mathrm{E} \Sigma_{n}^{r} \times X^{n} / \sim,
$$


with the equivalence-relation $\sim$ generated by the relations (i) and (ii) from 1.8 (with $e \in \mathrm{E} \Sigma_{n}^{r}$ ).

Notice that $\operatorname{sd}_{r} \Gamma^{+}(X)$ is in fact the bisimplicial set, which for $j$ fixed is the result of applying the subdivision functor to the cyclic set $\Gamma^{+}\left(X_{j}\right)$. The product 1.14 induces a monoid structure on $\operatorname{sd}_{r} \Gamma^{+}(X)$, and we let $\operatorname{sd}_{r} \Gamma(X)$ be the associated group completion: $\operatorname{sd}_{r} \Gamma(X)=\mathrm{Usd}_{r} \Gamma^{+}(X)$. Often we shall regard $\operatorname{sd}_{r} \Gamma^{+}(X)$ and $\operatorname{sd}_{r} \Gamma(X)$ simply as simplicial spaces, by restricting to the simplicial diagonal.

When $X$ is a $\mathrm{C}_{r}$-space we give $\operatorname{sd}_{r} \Gamma^{+}(X)$ a $\mathrm{C}_{r}$-action by letting $\mathrm{C}_{r}$ act diagonally on $\mathrm{E} \Sigma_{n}^{r} \times X^{n}$ in the obvious way. This is compatible with the monoid structure and induces a $\mathrm{C}_{r}$-action on $\operatorname{sd}_{r} \Gamma(X)$. The argument of [BE, 3.11] shows that $\operatorname{sd}_{r} \Gamma^{+}(X)$ is a free simplicial monoid on irreducible generators $[e, \mathbf{x}]$, where $e$ cannot be written as a direct $\operatorname{sum} e=e^{\prime} \oplus e^{\prime \prime}$, and where no component in $\mathbf{x}$ is the basepoint. This set of generators is preserved under the action of $\mathrm{C}_{r}$, so $\operatorname{sd}_{r} \Gamma^{+}(X)^{C_{s}}$ is a free submonoid, and

$$
\mathrm{U}\left[\operatorname{sd}_{r} \Gamma^{+}(X)^{\mathrm{C}_{s}}\right]=\operatorname{sd}_{r} \Gamma(X)^{\mathrm{C}_{s}} .
$$

Furthermore, it is not difficult to see that $\operatorname{sd}_{r} \Gamma^{+}(X)^{\mathrm{C}_{s}}$ is homotopy commutative, cf. [BE, 3.9].

Lemma 2.4. For a $\mathrm{C}_{r}$-space $X$ there is a natural equivariant homeomorphism

$$
\left|\Gamma^{+}(X)\right| \cong\left|\operatorname{sd}_{r} \Gamma^{+}(X)\right|,
$$

and similar for $\operatorname{sd}_{r} \Gamma(X)$.

Proof. This follows from naturality of the homeomorphism 2.2.

When studying the equivariant structure of $|\Gamma(X)|$ it is important to have a good understanding of the fixed-points. In particular we would like the $\mathrm{C}_{s^{-}}$ fixed-points of $\operatorname{sd}_{r} \Gamma^{+}(X)$ to be some quotient of

$$
\coprod_{n \geq 0}\left[\mathrm{E} \Sigma_{n}^{r} \times_{\Sigma_{n}} X^{n}\right]^{\mathrm{C}_{s}} .
$$

As a $\mathrm{C}_{s}$-space $\mathrm{E} \Sigma_{n}^{r}=\left(\mathrm{E} \Sigma_{n}^{r / s}\right)^{s}$. It follows from the definition of the actions that

$$
\left[e_{1}, \ldots, e_{s} ; x_{1}, \ldots, x_{n}\right] \in\left(\mathrm{E} \Sigma_{n}^{r / s}\right)^{s} \times_{\Sigma_{n}} X^{n}
$$

is fixed under the $\mathrm{C}_{s}$-action if and only if there exists an element $\gamma \in \Sigma_{n}$ with 


$$
\begin{array}{ll}
\text { (1) } & \gamma^{s}=1 \\
\text { (2) } & \left(e_{1}, \ldots, e_{s}\right)=\left(e_{1}, e_{1} \gamma, \ldots, e_{1} \gamma^{s-1}\right) \\
\text { (3) } & \left(x_{1}, \ldots, x_{n}\right)=\left(\mathrm{T} x_{\gamma(1)}, \ldots, \mathrm{T} x_{\gamma(n)}\right) .
\end{array}
$$

(Recall from section 2 that $\mathrm{T}$ is the generator for $\mathrm{C}_{r}$ ). We cannot impose relation 1.8 (ii) on the disjoint union 2.5 for the following reason: Given $(e, \mathbf{x}) \in \mathrm{E} \Sigma_{n} \times_{\Sigma_{n}} X^{n}$, fixed under the $\mathrm{C}_{s}$ action, and $\alpha \in \mathscr{M}(\mathbf{m}, \mathbf{n})$ which is entire for $\mathbf{x}$, it need not be true that

$$
\left(\alpha^{*}(e), \alpha^{*}(\mathbf{x})\right) \in \mathrm{E} \Sigma_{m}^{r} \times_{\Sigma_{m}} X^{m}
$$

is $\mathrm{C}_{s}$-fixed. However, if we choose $\gamma \in \Sigma_{n}$ satisfying (1), (2) and (3) above, and write it in cycle decomposition form with corresponding orbit decomposition $\mathbf{n}=J_{1} \coprod \ldots \coprod J_{k}$, we see from (3) that $x_{i}$ being the basepoint is a condition which is constant on the orbits $J_{\nu}$. We may therefore restrict our attention to morphisms $\alpha \in \mathscr{M}(\mathbf{m}, \mathbf{n})$ with $\alpha(\mathbf{m})$ a union of the orbits $J_{\nu}$, and from 1.3 we see that $\left(\alpha^{*}(e), \alpha^{*}(\mathbf{x})\right)$ is equal to

$$
\left(\alpha^{*}\left(e_{1}\right), \alpha^{*}\left(e_{1}\right) \circ \alpha^{*}(\gamma), \ldots, \alpha^{*}\left(e_{1}\right) \circ \alpha^{*}(\gamma)^{s-1}, x_{\alpha(1)}, \ldots, x_{\alpha(m)}\right)
$$

and therefore $\mathrm{C}_{s}$-fixed, cf. condition (1)-(3) above. In particular we may demand that $\alpha^{*}$ cancels all basepoints, and we get a relation on 2.5 as follows:

$$
\begin{aligned}
(e, \mathbf{x}) \sim\left(\alpha^{*}(e), \alpha^{*}(\mathbf{x})\right) & \text { for } \alpha \in \mathscr{M}(\mathbf{m}, \mathbf{n}) \text { satisfying } \\
& x_{i}=* \Leftrightarrow i \notin \alpha(\mathbf{m}) .
\end{aligned}
$$

One readily checks that this is independent of the choice of representative $(e, \mathbf{x})$.

LEMma 2.8. There is a natural isomorphism

$$
\operatorname{sd}_{r} \Gamma^{+}(X)^{\mathrm{C}_{s}}=\coprod_{n \geq 0}\left[\mathrm{E} \Sigma_{n}^{r} \times_{\Sigma_{n}} X^{n}\right]^{\mathrm{C}_{s}} / \sim,
$$

where on the right hand side we use the identifications 2.7.

The next lemma shows that in working with $\Gamma(X)$, we may always assume that $X$ is a Kan set.

Lemma 2.9. Let $X$ be a $\mathrm{C}_{r}$-space. The natural map $X \rightarrow \sin |X|$ induces an equivariant homotopy equivalence $|\Gamma(X)| \rightarrow|\Gamma(\sin |X|)|$.

Proof. By the equivariant Whitehead Theorem [A, 2.7], it is sufficient to show that the induced map on fixed points is an equivalence for every sub- 
group $\mathrm{C}_{s}$. According to Lemma 2.4 and Corollary 1.13, we may reduce the problem to considering

$$
\left|\operatorname{sd}_{s} \Gamma^{+}(X)^{\mathrm{C}_{s}}\right| \rightarrow\left|\operatorname{sd}_{s} \Gamma^{+}(\sin |X|)^{\mathrm{C}_{s}}\right| .
$$

In general, taking fixed points of a simplicial group action commutes with topological realization. The map $|X| \rightarrow|\sin | X||$ is therefore an equivariant homotopy-equivalence, again by the Whitehead Theorem and the wellknown non-equivariant case [May, 16.6]. The result now follows, since $\operatorname{sd}_{s} \Gamma^{+}$commutes with realization:

$$
\left|\operatorname{sd}_{s} \Gamma^{+}(X)^{\mathrm{C}_{s}}\right|=\coprod_{n \geq 0}\left[\left|\mathrm{E} \Sigma_{n}^{r}\right| \times_{\Sigma_{n}}|X|^{n}\right]^{\mathrm{C}_{s}} / \sim .
$$

\section{The Segal-tom Dieck splitting}

In this paragraph $r$ will be a fixed positive integer and $X$ will be a space with a left $\mathrm{C}_{r}$-action. For $s$ a divisor in $r$ we get a $\mathrm{C}_{r / s}$-action on $X^{\mathrm{C}_{s}}$ through the isomorphism $\mathrm{C}_{r} / C_{s} \cong \mathrm{C}_{r / s}$. We call $X$ equivariantly connected if $X^{\mathrm{C}_{s}}$ is connected for all subgroups in $\mathrm{C}_{r}$.

THEOREM 3.1.

(i) There is a simplicial map

$$
\Theta: \prod_{t \mid r} \Gamma^{+}\left(\mathrm{EC}_{r / t_{+}} \wedge_{\mathrm{C}_{r / t}} X^{\mathrm{C}_{t}}\right) \rightarrow \operatorname{sd}_{r} \Gamma^{+}(X)^{\mathrm{C}_{r}},
$$

natural in $X$ with respect to equivariant maps. Its realization is a homotopy multiplicative map of topological monoids and a homology isomorphism (integer coefficients).

(ii) There is a natural simplicial map

$$
\Theta: \prod_{t \mid r} \Gamma\left(\mathrm{EC}_{r / t_{+}} \wedge_{\mathrm{C}_{r / t}} X^{\mathrm{C}_{t}}\right) \rightarrow \operatorname{sd}_{r} \Gamma(X)^{\mathrm{C}_{r}}
$$

which induces a homotopy equivalence of realizations.

The proof of Theorem 3.1 occupies the rest of this section. First let us choose specific representatives for the conjugacy classes in $\Sigma_{n}$. Two elements in $\Sigma_{n}$ are conjugate if and only if they have the same cycle decomposition type. For a positive integer $s$ we consider the set $\mathscr{A}(s, n)$ of tuples $\boldsymbol{a}=\left(a_{t}\right)_{t \mid s}$ of natural numbers $a_{t} \geq 0$, indexed on the divisors in $s$ and satisfying $\sum_{t \mid s} a_{t} s / t=n$. To such an $\boldsymbol{a}$ we shall associate a permutation $\tilde{\boldsymbol{a}} \in \Sigma_{n}$ of type $\prod_{t \mid s}(s / t)^{a_{t}}$, that is, in the cycle decomposition of $\tilde{\boldsymbol{a}}$ there is exactly $a_{t}$ cycles of length $s / t$. (The reason for this choice of notation will be apparent from the 
definition of $\Theta$ below). Taking all choices of $\boldsymbol{a}$ will give us representatives for those conjugacy classes in $\Sigma_{n}$ in which the elements have orders dividing $s$. We define $\tilde{\boldsymbol{a}}$ by first dividing the set $\mathbf{n}$ into the number of blocks specified by $\boldsymbol{a}$, and then we let $\tilde{\boldsymbol{a}}$ act on each block by cyclic permutation. More explicitely, we first use lexicographical ordering to get a bijection $\left(a_{t}\right] \times(s / t] \cong\left(a_{t} s / t\right]$ and therefore an action of $a_{t}$ cycles of length $s / t$ on $\left(a_{t} s / t\right]$. Secondly, the usual ordering of the natural numbers induces an ordering of the divisors in $s$ and thus a bijection

$$
\mathbf{n}=(n] \cong \coprod_{t \mid s}\left(a_{t} s / t\right] \cong \coprod_{t \mid s}\left(a_{t}\right] \times(s / t],
$$

and $\tilde{\boldsymbol{a}}$ is defined by the induced action on $\mathbf{n}$. We also identify $\mathrm{C}_{t}$ with a subgroup of $\Sigma_{t}$ by choosing the $t$ cycle $(1, \ldots, t)$ as a generator.

Lemma 3.3. The centralizer $C_{\Sigma_{n}}(\tilde{\boldsymbol{a}})$ of $\tilde{\boldsymbol{a}}$ in $\Sigma_{n}$ is isomorphic to the product $\prod_{t \mid s} \Sigma_{a_{t}} \int \mathrm{C}_{s / t}$, where $\int$ denotes the wreath product (i.e. the semidirect product of $\Sigma_{a_{t}}$ and $\mathrm{C}_{s / t}^{a_{t}}$ ).

Proof. The isomorphism is given by associating to

$$
\prod_{t \mid s}\left(\sigma_{t}, c_{1}^{t}, \ldots, c_{a_{t}}^{t}\right) \in \prod_{t \mid s} \Sigma_{a_{t}} \int \mathrm{C}_{s / t}
$$

the element

$$
\oplus_{t \mid s}\left[\sigma_{t}\{s / t\} \circ\left(c_{1}^{t} \oplus \ldots \oplus c_{a_{t}}^{t}\right)\right] \in C_{\Sigma_{n}}(\boldsymbol{a}) .
$$

This notation should be interpreted as follows: Via the bijection 3.2, the sum $c_{1}^{t} \oplus \ldots \oplus c_{a_{t}}^{t}$ acts on $\left(a_{t}\right] \times(s / t]$ and $\sigma_{t}\{s / t\}$ permutes the $a_{t}$ blocks of length $s / t$.

For fixed $\boldsymbol{a}=\left(a_{t}\right)_{t \mid s}$ we define embeddings

$$
\chi_{t}^{s}:\left(X^{\mathrm{C}_{t}}\right)^{a_{t}} \rightarrow\left(X^{s / t}\right)^{a_{t}}, \quad \chi_{t}^{s}\left(x_{1}^{t}, \ldots, x_{a_{t}}^{t}\right)=\left(\mathbf{y}_{1}, \ldots, \mathbf{y}_{a_{t}}\right),
$$

where $\mathbf{y}_{\nu}=\left(\mathrm{T}^{(s / t)-1} x_{\nu}^{t}, \ldots, \mathrm{T} x_{\nu}^{t}, x_{\nu}^{t}\right)$ and $\mathrm{T}$ is the generator for $\mathrm{C}_{s}$. We also define

$$
\chi: \prod_{t \mid s}\left(X^{\mathrm{C}_{t}}\right)^{a_{t}} \rightarrow X^{n}, \quad \chi\left(\prod_{t \mid s} \mathbf{x}^{t}\right)=\prod_{t \mid s} \chi_{t}^{s}\left(\mathbf{x}^{t}\right)
$$

for $\mathbf{x}^{t} \in\left(X^{\mathrm{C}_{t}}\right)^{a_{t}}$. We convert the left $\mathrm{C}_{s / t}$-action on $X^{\mathrm{C}_{t}}$ to a right action by letting $x c=c^{-1} x$ for $x \in X^{\mathrm{C}_{t}}$ and $c \in \mathrm{C}_{s / t}$. There is also a right action of $\Sigma_{a_{t}}$ on $\left(X^{\mathrm{C}_{t}}\right)^{a_{t}}$ by permuting the coordinates, and by putting these two structures together we get a $\prod_{t \mid s} \Sigma_{a_{t}} \int \mathrm{C}_{s / t} \cong C_{\Sigma_{n}}(\tilde{\boldsymbol{a}})$ right action on $\prod_{t \mid s}\left(X^{\mathrm{C}_{t}}\right)^{a_{t}}$. As a 
subgroup of $\Sigma_{n}, C_{\Sigma_{n}}(\tilde{\boldsymbol{a}})$ also acts from the right on $X^{n}$, and it is not difficult to see that with these conventions $\chi$ becomes a $C_{\Sigma_{n}}(\tilde{\boldsymbol{a}})$-equivariant map.

Lemma 3.6. There is a natural isomorphism

$$
\psi: \coprod_{\boldsymbol{a} \in \mathscr{A}(s, n)} \mathrm{E} \Sigma_{n}^{r / s} \times_{C_{\Sigma_{n}}(\tilde{\boldsymbol{a}})} \prod_{t \mid s}\left(X^{\mathrm{C}_{t}}\right)^{a_{t}} \stackrel{\simeq}{\rightarrow}\left[\mathrm{E} \Sigma_{n}^{r} \times_{\Sigma_{n}} X^{n}\right]^{\mathrm{C}_{s}},
$$

which induces an isomorphism on quotient spaces

$$
\bar{\psi}: \bigvee_{\boldsymbol{a} \in \mathscr{A}(s, n)} \mathrm{E} \Sigma_{n}^{r / s}+\wedge_{C_{\Sigma_{n}}(\tilde{\boldsymbol{a}})} \bigwedge_{t \mid s}\left(X^{\mathrm{C}_{t}}\right)^{\left(a_{t}\right)} \stackrel{\simeq}{\rightarrow}\left[\mathrm{E} \Sigma_{n+}^{r} \wedge_{\Sigma_{n}} X^{(n)}\right]^{\mathrm{C}_{s}} .
$$

Here ( $k$ ) indicates $k$-fold smash product, and $\mathrm{E} \Sigma_{n}^{r / s}{ }_{+}=\mathrm{E} \Sigma_{n}^{r / s} \cup\{*\}$.

Proof. Given $\left(e, \prod_{t \mid s} \mathbf{x}^{t}, \boldsymbol{a}\right)$ in the domain of $\psi: \boldsymbol{a}=\left(a_{t}\right)_{t \mid s} \in \mathscr{A}(s, n)$, $e \in \mathrm{E} \Sigma_{n}^{r / s}$ and $\mathbf{x}^{t} \in\left(X^{\mathrm{C}_{t}}\right)^{a_{t}}$, we let

$$
\psi\left(e, \prod_{t \mid s} \mathbf{x}^{t}, \boldsymbol{a}\right)=\left(\left(e, e \tilde{\boldsymbol{a}}, \ldots, e \tilde{\boldsymbol{a}}^{s-1}\right), \chi\left(\prod_{t \mid s} \mathbf{x}^{t}\right)\right) .
$$

This is well-defined since $\chi$ is $C_{\Sigma_{n}}(\tilde{\boldsymbol{a}})$-equivariant, and from the description in 2.6 of the fixed-points of $\mathrm{E} \Sigma_{n}^{r} \times_{\Sigma_{n}} X^{n}$, it follows that the values of $\psi$ are $\mathrm{C}_{s}$-fixed.

To check surjectivity, assume we are given

$$
\left[e, e \gamma, \ldots, e \gamma^{s-1} ; \mathbf{x}\right] \in\left[\mathrm{E} \Sigma_{n}^{r} \times{ }_{\Sigma_{n}} X^{n}\right]^{\mathrm{C}_{s}}, \quad \text { with } \mathrm{Tx} \gamma=\mathbf{x}, \quad \text { cf. 2.6. }
$$

Now $\gamma$ is conjugate to some $\tilde{\boldsymbol{a}}$ induced from $\boldsymbol{a}=\left(a_{t}\right)_{t \mid s}$, say $\gamma=\tau \tilde{\boldsymbol{a}} \tau^{-1}$, and therefore

$$
\begin{aligned}
{\left[e, e \gamma, \ldots, e \gamma^{s-1} ; \mathbf{x}\right] } & =\left[e, e \tau \tilde{\boldsymbol{a}} \tau^{-1}, \ldots, e \tau \tilde{\boldsymbol{a}}^{s-1} \tau^{-1} ; \mathbf{x}\right] \\
& =\left[e \tau, e \tau \tilde{\boldsymbol{a}}, \ldots, e \tau \tilde{\boldsymbol{a}}^{s-1} ; \mathbf{x} \tau\right] .
\end{aligned}
$$

Since $\mathrm{T}(\mathbf{x} \tau) \tilde{\boldsymbol{a}}=\mathbf{x} \tau$ there exists $\mathbf{x}^{t} \in X^{\mathrm{C}_{t}}$ for $t \mid s$ such that $\chi\left(\prod_{t \mid s} \mathbf{x}^{t}\right)=\mathbf{x} \tau$. The proof of injectivity is just as easy and is left with the reader, as is the claim about $\bar{\psi}$.

To construct $\Theta$ we define for any $t$ dividing $r$ a homomorphism

$$
\Theta_{t}^{r}: \Gamma^{+}\left(\mathrm{EC}_{r / t_{+}} \wedge \mathrm{C}_{r / t} X^{\mathrm{C}_{t}}\right) \rightarrow \operatorname{sd}_{r} \Gamma^{+}(X)^{\mathrm{C}_{r}} .
$$

We keep $t$ fixed and consider a tuple $\boldsymbol{a}=\left(a_{u}\right)_{u \mid r} \in \mathscr{A}(r, n)$ with $a_{u}=0$ for $u \neq t$, and the associated element $\tilde{\boldsymbol{a}} \in \Sigma_{n}, n=a_{t} r / t$. For any space $Y$, $\operatorname{sd}_{r} \Gamma^{+}(Y)$ is obtained from $\bigsqcup_{n \geq 0} \mathrm{E} \Sigma_{n}^{r} \times_{\Sigma_{n}} Y^{n}$ by identifying the different summands according to cancelation of basepoints. We first ignore the basepoint-identifications, and consider the following string of maps. 


$$
\begin{aligned}
\widetilde{\Theta}_{t}^{r}: \mathrm{E} \Sigma_{a_{t}} \times \Sigma_{a_{t}}\left(\mathrm{EC}_{r / t} \times_{\mathrm{C}_{r / t}}\left(X^{\mathrm{C}_{t}}\right)\right)^{a_{t}} & \cong \mathrm{E}\left(\Sigma_{a_{t}} \int \mathrm{C}_{r / t}\right) \times_{\Sigma_{a_{t}}} \int \mathrm{C}_{r / t}\left(X^{\mathrm{C}_{t}}\right)^{a_{t}} \\
& \cong \mathrm{E} C_{\Sigma_{n}}(\tilde{\boldsymbol{a}}) \times_{C_{\Sigma_{n}}(\tilde{\boldsymbol{a}})}\left(X^{\mathrm{C}_{t}}\right)^{a_{t}} \\
& \stackrel{\mathrm{inc}}{\longrightarrow} \mathrm{E} \Sigma_{n} \times{ }_{C_{\Sigma_{n}}(\tilde{\boldsymbol{a}})}\left(X^{\mathrm{C}_{t}}\right)^{a_{t}} \\
& \stackrel{\psi}{\longrightarrow}\left(\mathrm{E} \Sigma_{n}^{r} \times_{\Sigma_{n}} X^{n}\right)^{\mathrm{C}_{r}}
\end{aligned}
$$

Explicitly,

$$
\begin{aligned}
\widetilde{\Theta}_{t}^{r}\left(e ; \prod_{\nu=1}^{a_{t}}\left(c_{\nu}, x_{\nu}\right)\right) & =\left(d, d \tilde{\boldsymbol{a}}, \ldots, d \tilde{\boldsymbol{a}}^{r-1} ; \chi_{t}^{r}\left(x_{1}, \ldots, x_{a_{t}}\right)\right), \quad \text { where } \\
d & =e\{r / t\} \circ\left(c_{1} \oplus \ldots \oplus c_{a_{t}}\right),
\end{aligned}
$$

cf. the proof of 3.3. Then $\Theta_{t}^{r}$ is obtained from $\widetilde{\Theta}_{t}^{r}$ by passage to quotient spaces.

Lemma 3.10. $\Theta_{t}^{r}$ is a homomorphism and induces a map of simplicial groups

$$
\Theta_{t}^{r}: \Gamma\left(\mathrm{EC}_{r / t_{+}} \wedge_{\mathrm{C}_{r / t}} X^{\mathrm{C}_{t}}\right) \rightarrow \operatorname{sd}_{r} \Gamma(X)^{\mathrm{C}_{r}} .
$$

Definition 3.11. The simplicial Segal-tom Dieck splitting

$$
\Theta: \prod_{t \mid r} \Gamma^{+}\left(\mathrm{EC}_{r / t_{+}} \wedge_{\mathrm{C}_{r / t}} X^{\mathrm{C}_{t}}\right) \rightarrow \mathrm{sd}_{r} \Gamma^{+}(X)^{\mathrm{C}_{r}}
$$

is the map that sends $\prod_{t \mid r} z_{t}$ into the product of the elements $\Theta_{t}^{r}\left(z_{t}\right)$ in the monoid $\operatorname{sd}_{r} \Gamma^{+}(X)^{\mathrm{C}_{r}}$ (using the natural order of the divisors in $r$ ). The same definition works with $\operatorname{sd}_{r} \Gamma$ instead of $\operatorname{sd}_{r} \Gamma^{+}$.

Proof of Theorem 3.1. To prove (i) we use the filtration of $\Gamma^{+}(X)$ by word length

$$
\{1\}=\Gamma^{+}(0)(X) \subseteq X=\Gamma^{+}(1)(X) \subseteq \ldots \subseteq \Gamma^{+}(n)(X) \subseteq \ldots \subseteq \Gamma^{+}(X),
$$

where

$$
\Gamma^{+}(n)(X)=\operatorname{Im}\left\{\coprod_{0 \leq i \leq n} \mathrm{E} \Sigma_{i} \times X^{i} \rightarrow \Gamma^{+}(X)\right\} \subseteq \Gamma^{+}(X) .
$$

This filtration has direct limit $\Gamma^{+}(X)$ and the filtration quotients are

$$
\Gamma^{+}(n)(X) / \Gamma^{+}(n-1)(X) \cong \mathrm{E} \Sigma_{n+} \wedge_{\Sigma_{n}} X^{(n)} .
$$

There is a similar filtration of $\operatorname{sd}_{r} \Gamma^{+}(X)$, and since the inclusions are equivariant, we obtain a filtration of $\operatorname{sd}_{r} \Gamma^{+}(X)^{\mathrm{C}_{r}}$ 


$$
\begin{aligned}
\{1\} & =\operatorname{sd}_{r} \Gamma^{+}(0)(X)^{\mathrm{C}_{r}} \subseteq X^{\mathrm{C}_{r}} \\
& =\operatorname{sd}_{r} \Gamma^{+}(1)(X)^{\mathrm{C}_{r}} \subseteq \ldots \subseteq \operatorname{sd}_{r} \Gamma^{+}(n)(X)^{\mathrm{C}_{r}} \subseteq \ldots
\end{aligned}
$$

with filtration quotients

$$
\operatorname{sd}_{r} \Gamma^{+}(n)(X)^{\mathrm{C}_{r}} / \mathrm{sd}_{r} \Gamma^{+}(n-1)(X)^{\mathrm{C}_{r}} \cong\left[\mathrm{E} \Sigma_{n+}^{r} \wedge_{\Sigma_{n}} X^{(n)}\right]^{\mathrm{C}_{r}} .
$$

We also filter the domain of $\Theta$ with subspaces $F_{n}$ equal to

$$
\operatorname{Im}\left\{\coprod_{\substack{a \in \mathcal{S}(r, m) \\ m \leq n}} \prod_{t \mid r} \mathrm{E} \Sigma_{a_{t}} \times\left(\mathrm{EC}_{r / t_{+}} \wedge_{\mathrm{C}_{r / t}} X^{\mathrm{C}_{t}}\right)^{a_{t}} \rightarrow \prod_{t \mid r} \Gamma^{+}\left(\mathrm{EC}_{r / t_{+}} \wedge_{\mathrm{C}_{r / t}} X^{\mathrm{C}_{t}}\right)\right\}
$$

and

$$
F_{n} / F_{n-1}=\bigvee_{a \in \mathscr{A}(r, n)} \bigwedge_{t \mid r} \mathrm{E} \Sigma_{a_{t+}} \wedge_{\Sigma_{a_{t}}}\left(\mathrm{EC}_{r / t_{+}} \wedge_{\mathrm{C}_{r / t}} X^{\mathrm{C}_{t}}\right)^{\left(a_{t}\right)}
$$

By construction $\Theta$ is a filtration preserving map, and it suffices to show that $\Theta$ induces a homology equivalence on the filtration quotients. Indeed, we have spectral-sequences associated with the filtrations of domain and target with $\mathrm{E}^{1}$-terms the relative homology groups of the filtration quotients. From the definition 3.8 we get

$$
\begin{aligned}
& \Theta: \bigvee_{\mathscr{A}(r, n)} \bigwedge_{t \mid r} \mathrm{E} \Sigma_{a_{t+}} \wedge_{\Sigma_{a_{t}}}\left(\mathrm{EC}_{r / t_{+}} \wedge_{\mathrm{C}_{r / t}} X^{\mathrm{C}_{t}}\right)^{\left(a_{t}\right)} \\
& \longrightarrow \bigvee_{\mathscr{A}(r, n)} \mathrm{E}_{\Sigma_{\Sigma_{n}}(\tilde{\boldsymbol{a}})_{+}} \wedge_{C_{\Sigma_{n}}(\tilde{a})} \bigwedge_{t \mid r}\left(X^{\mathrm{C}_{t}}\right)^{\left(a_{t}\right)} \\
& \stackrel{\text { inc }}{\longrightarrow} \bigvee_{\mathscr{A}(r, n)} \mathrm{E} \Sigma_{n+} \wedge_{\Sigma_{\Sigma_{n}}(\tilde{a})} \bigwedge_{t \mid r}\left(X^{\mathrm{C}_{t}}\right)^{\left(a_{t}\right)} \\
& \stackrel{\bar{\psi}}{\longrightarrow}\left[\mathrm{E} \Sigma_{n+}^{r} \wedge_{\Sigma_{n}} X^{n}\right]^{\mathrm{C}_{r}} .
\end{aligned}
$$

Here the first map is an isomorphism by definition, the second an equivalence since $\mathrm{E} C_{\Sigma_{n}}(\tilde{\boldsymbol{a}}) \rightarrow \mathrm{E} \Sigma_{n}$ is a $C_{\Sigma_{n}}(\tilde{\boldsymbol{a}})$-equivariant equivalence, and finally $\bar{\psi}$ is an isomorphism by Lemma 3.6.

That $\Theta$ is homotopy multiplicative follows because $\operatorname{sd}_{r} \Gamma^{+}(X)$ is homotopy commutative. To prove (ii) we show that $\Theta$ induces an isomorphism on homology. This follows from Corollary 1.11. Indeed, for any homotopycommutative simplicial monoid $M, \mathrm{H}_{*}(M) \otimes_{\mathbf{Z}_{\left[\pi_{0}(M)\right]}} \mathrm{Z}\left[\pi_{0}(\mathrm{U}(M))\right]$ is the localization of $\mathrm{H}_{*}(M)$ at the multiplicative subset $\pi_{0}(M)$.

Corollary 3.14. The natural inclusion $\left|\Gamma^{+}(X)\right| \rightarrow|\Gamma(X)|$ is a $\mathrm{C}_{r}$-equivariant homotopy-equivalence, when $X$ is equivariantly connected. 
Proof. Use of the Whitehead Theorem and Lemma 2.4 reduces us to showing that $\left|\operatorname{sd}_{s} \Gamma^{+}(X)^{\mathrm{C}_{s}}\right| \rightarrow\left|\operatorname{sd}_{s} \Gamma(X)^{\mathrm{C}_{s}}\right|$ is an equivalence for every subgroup $\mathrm{C}_{s}$. By Theorem 3.1, $\operatorname{sd}_{s} \Gamma^{+}(X)^{\mathrm{C}_{s}}$ is a connected free simplicial mono$\mathrm{id}$, and because $\operatorname{sd}_{s} \Gamma(X)^{\mathrm{C}_{s}}=\mathrm{Usd}_{s} \Gamma^{+}(X)^{\mathrm{C}_{s}}$, the result follows from 1.10 since a homology isomorphism of connected $\mathrm{H}$-spaces is a homotopy equivalence.

\section{Cofibration sequences}

We call a sequence of pointed spaces $F \rightarrow E \stackrel{f}{\rightarrow} B$ a homotopy fibration if the map from $F$ to the homotopy fiber of $f$ is a homotopy equivalence. In [BE, 7.4] it is proved that $\Gamma$ turns cofibration sequences into homotopy fibrations. We prove an equivariant analogue of this.

Proposition 4.1. Let $B \subseteq A$ be a pair of $\mathrm{C}_{r}$-spaces, and let $q: A \rightarrow A / B$ be the quotient map. Then the natural map

$$
\operatorname{sd}_{r} \Gamma(B)^{\mathrm{C}_{r}} \rightarrow \operatorname{Ker}\left\{\operatorname{sd}_{r} \Gamma q: \operatorname{sd}_{r} \Gamma(A)^{\mathrm{C}_{r}} \rightarrow \operatorname{sd}_{r} \Gamma(A / B)^{\mathrm{C}_{r}}\right\}
$$

is an equivalence. Consequently there is a homotopy fibration sequence

$$
\mathrm{sd}_{r} \Gamma(B)^{\mathrm{C}_{r}} \rightarrow \mathrm{sd}_{r} \Gamma(A)^{\mathrm{C}_{r}} \rightarrow \mathrm{sd}_{r} \Gamma(A / B)^{\mathrm{C}_{r}} .
$$

Proof. The degree-wise construction $\operatorname{sd}_{r} \Gamma(\cdot)$ is a functor from $\mathrm{C}_{r}$-spaces to simplicial groups, and Lemma 7.2 of [BE] easily generalizes to $\mathrm{C}_{r}$-spaces. Thus it suffices to prove that

$$
i_{1} \times i_{2}: \operatorname{sd}_{r} \Gamma\left(A_{1}\right)^{\mathrm{C}_{r}} \times \operatorname{sd}_{r} \Gamma\left(A_{2}\right)^{\mathrm{C}_{r}} \rightarrow \operatorname{sd}_{r} \Gamma\left(A_{1} \vee A_{2}\right)^{\mathrm{C}_{r}}
$$

is an equivalence for any pair of $\mathrm{C}_{r}$-spaces, or equivalently that the natural monoid homomorphism

$$
p_{1} \times p_{2}: \operatorname{sd}_{r} \Gamma\left(A_{1} \vee A_{2}\right)^{\mathrm{C}_{r}} \rightarrow \operatorname{sd}_{r} \Gamma\left(A_{1}\right)^{\mathrm{C}_{r}} \times \operatorname{sd}_{r} \Gamma\left(A_{2}\right)^{\mathrm{C}_{r}}
$$

is an equivalence. We consider the diagram

$$
\begin{aligned}
& \prod_{t \mid r} \Gamma^{+}\left(\mathrm{EC}_{r / t+\wedge_{\mathrm{C}_{r / t}}}\left(A_{1} \vee A_{2}\right)^{\mathrm{C}_{t}}\right) \stackrel{\Theta}{\longrightarrow} \quad \operatorname{sd}_{r} \Gamma^{+}\left(A_{1} \vee A_{2}\right)^{\mathrm{C}_{r}} \\
& \downarrow \downarrow \\
& \prod_{t \mid r} \Gamma^{+}\left(Z_{1}^{t}\right) \times \prod_{t \mid r} \Gamma^{+}\left(Z_{2}^{t}\right) \quad \stackrel{\Theta \times \Theta}{\longrightarrow} \operatorname{sd}_{r} \Gamma^{+}\left(A_{1}\right)^{\mathrm{C}_{r}} \times \operatorname{sd}_{r} \Gamma^{+}\left(A_{2}\right)^{\mathrm{C}_{r}},
\end{aligned}
$$

where we write $Z_{i}^{t}=\mathrm{EC}_{r / t_{+}} \wedge_{\mathrm{C}_{r / t}} A_{i}^{\mathrm{C}_{t}}$, for $i=1,2$. The left vertical map is a homology equivalence by the non-equivariant version of the lemma cf. [BE, 7.5], and the horizontal maps are homology equivalences by Theorem 3.1. 
Therefore the right vertical map is also a homology equivalence, and it now follows from Corollary 1.13 that 4.3 is a homotopy equivalence.

Corollary 4.4. Let $X$ be a $\mathrm{C}_{r}$-space. Then the natural map

$$
\left|\operatorname{sd}_{r} \Gamma(X)\right|^{\mathrm{C}_{r}} \rightarrow \Omega\left(\left|\operatorname{sd}_{r} \Gamma\left(S^{1} \wedge X\right)\right|\right)^{\mathrm{C}_{r}}
$$

is a homotopy equivalence.

Proof. Consider the equivariant cofibration sequence

$$
X \rightarrow \mathrm{C} X \rightarrow \Sigma X,
$$

where $\mathrm{C} X=\mathrm{I} \wedge X$ is the reduced cone on $X$, equivariantly contractible by a simplicial homotopy. By Proposition 4.1 there is a fibration sequence

$$
\left|\operatorname{sd}_{r} \Gamma(X)\right|^{\mathrm{C}_{r}} \rightarrow\left|\operatorname{sd}_{r} \Gamma(\mathrm{C} X)\right|^{\mathrm{C}_{r}} \rightarrow\left|\operatorname{sd}_{r} \Gamma(\Sigma X)\right|^{\mathrm{C}_{r}} .
$$

The claim now follows by comparing this with the path-space fibration, since the standard contracting homotopy $\mathrm{I} \wedge \mathrm{I} \rightarrow \mathrm{I}$ induces a map $\beta$ that fits in the diagram



\section{The R-map}

In this section we define the restriction map

$$
\mathrm{R}: \mathrm{sd}_{r} \Gamma(X)^{\mathrm{C}_{s}} \rightarrow \operatorname{sd}_{r / s} \Gamma\left(X^{\mathrm{C}_{s}}\right) .
$$

Again we first ignore the basepoint-identifications in $\operatorname{sd}_{r} \Gamma^{+}(X)^{\mathrm{C}_{s}}$, and assume we are given an element

$$
[e ; \mathbf{x}] \in\left[\mathrm{E} \Sigma_{n}^{r} \times_{\Sigma_{n}} X^{n}\right]^{\mathrm{C}_{s}} .
$$

We write $e=\left(e_{1}, \ldots, e_{s}\right) \in\left(\mathrm{E} \Sigma_{n}^{r / s}\right)^{s}$ and consider the set

$$
\mathscr{S}=\left\{u \in \mathbf{n}: e_{1}(u)=\ldots=e_{s}(u)\right\} .
$$

(This makes sense, since in simplicial degree $i, \mathrm{E}_{i} \Sigma_{n}^{r / s}=\Sigma_{n}^{(r / s)(i+1)}$.) Let $m=|\mathscr{S}|$ and let $\alpha: \mathbf{m} \rightarrow \mathbf{n}$ be the strictly increasing map with 
$\alpha(\mathbf{m})=\mathscr{S} \subseteq \mathbf{n}$. It is easy to see that $\alpha^{*}\left(e_{1}\right)=\ldots=\alpha^{*}\left(e_{s}\right) \in \mathrm{E} \Sigma_{m}^{r / s}$, and we check below that $\alpha^{*}(\mathbf{x}) \in\left(X^{\mathrm{C}_{s}}\right)^{m}$. Hence we may define

$$
\mathrm{R}([e ; \mathbf{x}])=\left[\alpha^{*}\left(e_{1}\right) ; \alpha^{*}(\mathbf{x})\right] \in \mathrm{E} \Sigma_{m}^{r / s} \times_{\Sigma_{m}}\left(X^{\mathrm{C}_{s}}\right)^{m} .
$$

Lemma 5.1. The above construction gives a well-defined $\mathrm{C}_{r} / \mathrm{C}_{s} \cong \mathrm{C}_{r / s}$ equivariant map

$$
\mathrm{R}: \mathrm{sd}_{r} \Gamma^{+}(X)^{\mathrm{C}_{s}} \rightarrow \mathrm{sd}_{r / s} \Gamma^{+}\left(X^{\mathrm{C}_{s}}\right) .
$$

This is a homomorphism of simplicial monoids, and it induces

$$
\mathrm{R}: \mathrm{sd}_{r} \Gamma(X)^{\mathrm{C}_{s}} \rightarrow \mathrm{sd}_{r / s} \Gamma\left(X^{\mathrm{C}_{s}}\right) .
$$

Proof. To check that $\mathrm{R}$ is well-defined we consider the diagram

$$
\begin{aligned}
& \coprod_{\substack{a \in \mathscr{A}(s, n) \\
n \geq 0}} \mathrm{E} \Sigma_{n}^{r / s} \times_{C_{\Sigma_{n}}(\tilde{\boldsymbol{a}})} \prod_{t \mid s}\left(X^{\mathrm{C}_{t}}\right)^{a_{t}} \stackrel{\psi}{\longrightarrow} \coprod_{n \geq 0}\left[\mathrm{E} \Sigma_{n}^{r} \times \Sigma_{n} X^{n}\right]^{\mathrm{C}_{s}} \\
& \downarrow R^{\prime} \quad \downarrow R \\
& \coprod_{m \geq 0} \mathrm{E} \Sigma_{m}^{r / s} \times{ }_{\Sigma_{m}}\left(X^{\mathrm{C}_{s}}\right)^{m}=\coprod_{m \geq 0} \mathrm{E} \Sigma_{m}^{r / s} \times{ }_{\Sigma_{m}}\left(X^{\mathrm{C}_{s}}\right)^{m},
\end{aligned}
$$

where $\mathrm{R}^{\prime}$ is given as follows. Fix the component corresponding to $\boldsymbol{a} \in \mathscr{A}(s, n)$, and let $\alpha \in \mathscr{M}\left(\left(a_{s}\right], \mathbf{n}\right)$ be such that $\alpha\left(\left(a_{s}\right]\right) \subseteq \mathbf{n}$ corresponds to $\left(a_{s}\right] \times(1]$ in the decomposition $\mathbf{n} \cong \coprod_{t \mid s}\left(a_{t}\right] \times(s / t]$, cf. 3.2. Then

$$
\mathrm{R}^{\prime}: \mathrm{E} \Sigma_{n}^{r / s} \times{ }_{C_{\Sigma_{n}}(\tilde{\boldsymbol{a}})} \prod_{t \mid r}\left(X^{\mathrm{C}_{t}}\right)^{a_{t}} \rightarrow \mathrm{E} \Sigma_{a_{s}}^{r / s} \times \Sigma_{\Sigma_{a_{s}}}\left(X^{\mathrm{C}_{s}}\right)^{a_{s}}
$$

is given by $\mathrm{R}^{\prime}\left(e, \prod_{t \mid s} \mathbf{x}^{t}\right)=\left(\alpha^{*} e, \mathbf{x}^{s}\right)$. Using this, it is not difficult to see that $\mathrm{R}$ respects the basepoint-identifications.

We next show that the $\mathrm{R}$ maps are compatible with the Segal-tom Dieck splitting 3.1.

Proposition 5.3. There is a commutative diagram

$$
\begin{array}{ccc}
\operatorname{sd}_{r} \Gamma(X)^{\mathrm{C}_{r}} & \stackrel{\mathrm{R}}{\longrightarrow} & \operatorname{sd}_{r / s} \Gamma\left(X^{\mathrm{C}_{s}}\right)^{\mathrm{C}_{r / s}} \\
\Theta \uparrow & \uparrow \Theta \\
\prod_{t \mid r} \Gamma\left(\mathrm{EC}_{r / t_{+}} \wedge_{\mathrm{C}_{r / t}} X^{\mathrm{C}_{t}}\right) & \stackrel{\text { proj }}{\longrightarrow} & \prod_{u \mid(r / s)} \Gamma\left(\mathrm{EC}_{(r / s u)_{+}} \wedge_{\mathrm{C}_{(r / s u)}}\left[X^{\left.\mathrm{C}_{s}\right]} \mathrm{C}_{u}\right),\right.
\end{array}
$$

where proj maps the component indexed by $t$ to the component indexed by $u=t / s$ when $s$ divides $t$, and to the basepoint otherwise. This gives a split homotopy fibration 


$$
\prod_{t \mid r, s \nmid t} \Gamma\left(\mathrm{EC}_{r / t_{+}} \wedge_{\mathrm{C}_{r / t}} X^{\mathrm{C}_{t}}\right) \rightarrow \mathrm{sd}_{r} \Gamma(X)^{\mathrm{C}_{r}} \stackrel{\mathrm{R}}{\longrightarrow} \mathrm{sd}_{r / s} \Gamma\left(X^{\mathrm{C}_{s}}\right)^{\mathrm{C}_{r / s}} .
$$

If in particular $\mathrm{C}_{r}$ acts freely on $X$ away from the basepoint, we get an equivalence

$$
\Gamma\left(X / \mathrm{C}_{r}\right) \simeq \operatorname{sd}_{r} \Gamma(X)^{\mathrm{C}_{r}}
$$

Proof. The $\mathrm{R}$ map is a homomorphism, so to check commutativity we may restrict the attention to one factor $\Gamma\left(\mathrm{EC}_{r / t_{+}} \wedge_{\mathrm{C}_{r / t}} X^{\mathrm{C}_{t}}\right)$ corresponding to a fixed $t$. Also, by functoriality it suffices then to consider $\operatorname{sd}_{r} \Gamma^{+}$instead of $\operatorname{sd}_{r} \Gamma$. With the notation from 3.9 the value of $\Theta$ on an element

$$
z=\left[e ; \prod_{\nu=1}^{a_{t}}\left(c_{\nu}, x_{\nu}\right)\right] \in \Gamma^{+}\left(\mathrm{EC}_{r / t_{+}} \wedge_{\mathrm{C}_{r / t}} X^{\mathrm{C}_{t}}\right)
$$

is given by

$$
\Theta_{t}^{r}(z)=\left(d, d \tilde{\boldsymbol{a}}, \ldots, d \tilde{\boldsymbol{a}}^{r-1} ; \chi_{t}^{r}\left(x_{1}, \ldots, x_{a_{t}}\right)\right),
$$

where $\tilde{\boldsymbol{a}}$ has type $(r / t)^{a_{t}}$. It follows from the definition that the effect of $\mathrm{R}$ on $\Theta_{t}^{r}(z)$ depend on whether or not $\tilde{\boldsymbol{a}}^{r / s}=1$, or equivalently whether or not $s$ divides $t$. When this is the case then obviously $\operatorname{R}_{t}^{r}(z)=\Theta_{r / s}^{t / s}(z)$, and when $s \nmid t$, R maps $\Theta_{t}^{r}(z)$ to the basepoint. This proves commutativity.

The next result will be used in the proof of Proposition 7.1. By induction it allows us to reduce problems about $\operatorname{sd}_{r} \Gamma(X)^{\mathrm{C}_{r}}$ to the case where $X$ is $\mathrm{C}_{p}$-free for some prime $p$ dividing $r$. Thus let $p$ be a prime divisor in $r$ and let $\mathrm{C}_{q} \subseteq \mathrm{C}_{r}$ be the Sylow- $p$ subgroup. We let $\mathrm{C}_{r}$ act on $\mathrm{C}_{q}$ through the quotient map $\mathrm{C}_{r} \rightarrow \mathrm{C}_{r} / \mathrm{C}_{r / q} \cong \mathrm{C}_{q}$. This action is $\mathrm{C}_{p}$-free and trivial for subgroups $\mathrm{C}_{s}$ with $s$ prime to $p$.

COROllary 5.5. The $\mathrm{C}_{r}$-equivariant projection $\mathrm{EC}_{q_{+}} \wedge X \rightarrow X$ fits into a split homotopy fibration sequence:

$$
\operatorname{sd}_{r} \Gamma\left(\mathrm{EC}_{q_{+}} \wedge X\right)^{\mathrm{C}_{r}} \rightarrow \mathrm{sd}_{r} \Gamma(X)^{\mathrm{C}_{r}} \stackrel{\mathrm{R}}{\longrightarrow} \operatorname{sd}_{r / p} \Gamma\left(X^{\mathrm{C}_{p}}\right)^{\mathrm{C}_{r / p}} .
$$

Proof. Notice first that the map $\mathrm{EC}_{q_{+}} \wedge X \rightarrow X$ is a non-equivariant homotopy equivalence. The corollary then follows by applying the homotopy fibration (5.4) with $s=p$ to the spaces $X$ and $\mathrm{EC}_{q_{+}} \wedge X$.

\section{The Wirthmüller Isomorphism}

Let $\mathrm{C}_{s} \subseteq \mathrm{C}_{r}$ be a pair of cyclic groups, and let $X$ be a $\mathrm{C}_{s}$-space. Then the $\mathrm{C}_{s^{-}}$ equivariant projection 


$$
\mathscr{W}: \mathrm{C}_{r+} \wedge_{\mathrm{C}_{s}} X \rightarrow X, \quad \mathscr{W}(c, x)= \begin{cases}c x, & \text { for } c \in \mathrm{C}_{s} \\ *, & \text { otherwise }\end{cases}
$$

induces a homotopy equivalence (the Wirthmüller isomorphism)

$$
\mathscr{W}: \mathrm{Q}_{\mathrm{C}_{\mathrm{r}}}\left(\mathrm{C}_{r+} \wedge_{\mathrm{C}_{s}} X\right)^{\mathrm{C}_{r}} \rightarrow \mathrm{Q}_{\mathrm{C}_{s}}(X)^{\mathrm{C}_{s}},
$$

cf. $[\mathrm{A}, 5.2]$. We shall prove a similar result for our model $\Gamma$.

Proposition 6.3. The projection 6.1 induces a homotopy equivalence

$$
\mathscr{W}: \operatorname{sd}_{r} \Gamma\left(\mathrm{C}_{r+} \wedge_{\mathrm{C}_{s}} X\right)^{\mathrm{C}_{r}} \rightarrow \operatorname{sd}_{r} \Gamma(X)^{\mathrm{C}_{s}} .
$$

For the proof we shall need in our model an analogue of the forgetful map

$$
\mathrm{Q}_{\mathrm{C}_{\mathrm{r}}}(X) \rightarrow \mathrm{Q}_{\mathrm{C}_{s}}(X),
$$

that regards a $\mathrm{C}_{r}$-representation simply as a $\mathrm{C}_{s}$-representation. This is supplied by the following lemma.

Lemma 6.4. There is a $\mathrm{C}_{s}$-equivariant map

$$
\rho: \operatorname{sd}_{r} \Gamma(X) \rightarrow \operatorname{sd}_{s} \Gamma(X),
$$

inducing a homotopy equivalence

$$
\operatorname{sd}_{r} \Gamma(X)^{\mathrm{C}_{s}} \rightarrow \operatorname{sd}_{s} \Gamma(X)^{\mathrm{C}_{s}} .
$$

Proof. As a $\mathrm{C}_{s}$-space $\mathrm{E} \Sigma_{n}^{r} \cong\left(\mathrm{E} \Sigma_{n}^{r / s}\right)^{s}$ and we get a $\mathrm{C}_{s}$-map

$$
\left(\mathrm{E} \Sigma_{n}^{r / s}\right)^{s} \times_{\Sigma_{n}} X^{n} \rightarrow\left(\mathrm{E} \Sigma_{n}\right)^{s} \times_{\Sigma_{n}} X^{n}
$$

by projecting $\mathrm{E} \Sigma_{n}^{r / s}$ on, say, the first factor. Clearly this induces a monoid homomorphism $\operatorname{sd}_{r} \Gamma^{+}(X) \rightarrow \operatorname{sd}_{s} \Gamma^{+}(X)$, and $\rho$ is induced from this by the functor U. To see that it is an equivalence on fixed points, we use the filtration 3.12 and the proof follows from Lemma 3.6 and the fact that the projection $\mathrm{E} \Sigma_{n}^{r / s} \rightarrow \mathrm{E} \Sigma_{n}$ is a $\Sigma_{n}$-equivariant equivalence.

Proof of Proposition 6.3. The Segal-tom Dieck splitting 3.1 shows that

$$
\operatorname{sd}_{r} \Gamma\left(\mathrm{C}_{r+} \wedge_{\mathrm{C}_{s}} X\right)^{\mathrm{C}_{r}} \cong \prod_{t \mid s} \Gamma\left(\mathrm{EC}_{r / t_{+}} \wedge_{\mathrm{C}_{r / t}}\left(\mathrm{C}_{r+} \wedge_{\mathrm{C}_{s}} X\right)^{\mathrm{C}_{t}}\right) .
$$

For $t$ dividing $s$ we denote by $\check{c}$ the image of $c \in \mathrm{EC}_{r / s}$ under the inclusion $\mathrm{EC}_{s / t} \rightarrow \mathrm{EC}_{r / t} \subseteq \mathrm{E} \Sigma_{r / t}$. The map

$$
i: \mathrm{EC}_{s / t_{+}} \wedge_{\mathrm{C}_{s / t}} X^{\mathrm{C}_{t}} \rightarrow \mathrm{EC}_{r / t_{+}} \wedge_{\mathrm{C}_{r / t}}\left(\mathrm{C}_{r+} \wedge_{\mathrm{C}_{s}} X\right)^{\mathrm{C}_{t}}, \quad(c, x) \mapsto(\check{\mathrm{c}}, 1, x)
$$


is an equivalence, and the proposition thus follows, if we prove commutativity in the diagram

$$
\begin{array}{ccc}
\prod_{t \mid s} \Gamma\left(\mathrm{EC}_{r / t_{+}} \wedge_{\mathrm{C}_{r / t}}\left(\mathrm{C}_{r+} \wedge_{\mathrm{C}_{s}} X\right)^{\left.\mathrm{C}_{t}\right)}\right. & \stackrel{\Theta}{\longrightarrow} & \operatorname{sd}_{r} \Gamma\left(\mathrm{C}_{r+} \wedge_{\mathrm{C}_{s}} X\right)^{\mathrm{C}_{r}} \\
{ }_{i \uparrow} \uparrow & \downarrow \rho \mathscr{W} \\
\prod_{t \mid s} \Gamma\left(\mathrm{EC}_{s / t_{+}} \wedge_{\mathrm{C}_{s / t}} X^{\mathrm{C}_{t}}\right) & \stackrel{\Theta}{\longrightarrow} & \operatorname{sd}_{s} \Gamma(X)^{\mathrm{C}_{s}} .
\end{array}
$$

Since both $\mathscr{W}$ and $\rho$ are monoid homomorphisms, we may fix $t$ dividing $s$ and consider

$$
z=\left[e ; \prod_{\nu=1}^{a_{t}}\left(c_{\nu}, x_{\nu}\right)\right] \in \Gamma^{+}\left(\mathrm{EC}_{s / t_{+}} \wedge_{\mathrm{C}_{s / t}} X^{\mathrm{C}_{t}}\right) .
$$

We calculate

$$
\begin{aligned}
& \rho \mathscr{W} \Theta_{t}^{r} i(z)=\left[\left(d, d \tilde{\boldsymbol{a}}^{r / s}, \ldots, d \tilde{\boldsymbol{a}}^{(r / s)(s-1)}\right) ;\right. \\
& \left.\mathscr{W} \chi_{r}^{t}\left(\prod_{\nu=1}^{a_{t}}\left(1, x_{\nu}\right)\right)\right] \in \operatorname{sd}_{s} \Gamma(X)^{\mathrm{C}_{s}},
\end{aligned}
$$

where $n=a_{t}(r / t), \tilde{\boldsymbol{a}} \in \Sigma_{n}$ has type $(r / t)^{a_{t}}$ and $d=e\{r / t\} \circ\left(\check{c}_{1} \oplus \ldots \oplus \check{c}_{a}\right)$.

Recall from 3.4 that if $\mathrm{T}$ denotes the generator for $\mathrm{C}_{r}$ then

$$
\begin{gathered}
\chi_{t}^{r}:\left(\left(\mathrm{C}_{r+} \wedge_{\mathrm{C}_{s}} X\right)^{\mathrm{C}_{t}}\right)^{a_{t}} \rightarrow\left(\mathrm{C}_{r+} \wedge_{\mathrm{C}_{s}} X\right)^{n}, \quad \text { has } \\
\chi_{t}^{r}\left(\prod_{\nu=1}^{a_{t}}\left(1, x_{\nu}\right)\right)=\left(\mathbf{y}_{1}, \ldots, \mathbf{y}_{a_{t}}\right)
\end{gathered}
$$

with $\mathbf{y}_{\nu}=\left(\left(\mathrm{T}^{(r / t)-1}, x_{\nu}\right), \ldots,\left(\mathrm{T}, x_{\nu}\right),\left(1, x_{\nu}\right)\right)$. Similarly, since $\mathrm{C}_{s}$ is generated by $\mathrm{T}^{r / s}$ we have

$$
\chi_{t}^{s}:\left(X^{\mathrm{C}_{t}}\right)^{a_{t}} \rightarrow X^{m}, \quad \chi_{t}^{s}\left(x_{1}, \ldots, x_{a_{t}}\right)=\left(\mathbf{z}_{1}, \ldots, \mathbf{z}_{a_{t}}\right),
$$

where $\quad m=a_{t}(s / t) \quad$ and $\quad \mathbf{z}_{\nu}=\left(\mathrm{T}^{(r / s)(s / t-1)} x_{\nu}, \ldots, \mathrm{T}^{(r / s)} x_{\nu}, x_{\nu}\right) . \quad$ Define $\beta \in \mathscr{M}(\mathbf{m}, \mathbf{n})$ by commutativity of the diagram

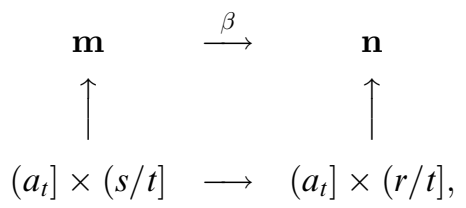

where the lower horizontal map sends $(\nu, u)$ to $(\nu, u(r / s))$. Now $\mathrm{T}^{u} \in \mathrm{C}_{s}$ precisely if $r / s$ divides $u$, and therefore

$$
\beta^{*}\left(\mathscr{W} \chi_{t}^{r}\left(\left(1, x_{1}\right), \ldots,\left(1, x_{a}\right)\right)\right)=\chi_{t}^{s}\left(x_{1}, \ldots, x_{a}\right)
$$

and 6.5 reduces to 


$$
\left[\beta^{*}(d), \beta^{*}\left(d \tilde{\boldsymbol{a}}^{r / s}\right), \ldots, \beta^{*}\left(d \tilde{\boldsymbol{a}}^{(r / s)(s-1)}\right) ; \chi_{t}^{s}\left(x_{1}, \ldots, x_{a}\right)\right] .
$$

To prove 6.6 equal to $\Theta_{t}^{s}\left(\left[e ; \prod_{\nu=1}^{a_{t}}\left(c_{\nu}, x_{\nu}\right)\right]\right)$ we just have to observe that $\boldsymbol{b}=\beta^{*}\left(\tilde{\boldsymbol{a}}^{r / s}\right)$ has type $(s / t)^{a_{t}}$, and to verify the easy formulas

$$
\begin{array}{r}
\beta^{*}(d)=e\{s / t\} \circ\left(c_{1} \oplus \ldots \oplus c_{a}\right), \quad \text { and } \\
\beta^{*}\left(d \tilde{\boldsymbol{a}}^{u(r / s)}\right)=\beta^{*}(d) \boldsymbol{b}^{u}, \quad \text { for } u=1, \ldots, s-1 .
\end{array}
$$

\section{Comparison with equivariant infinite loop spaces}

Let $\mathscr{R}=\mathrm{R}\left[\mathrm{C}_{r}\right]$. We identify $S^{l \mathscr{R}} \cong\left(S^{l}\right)^{(r)}$, where the action on the right hand side is cyclic permutation of the $r$ factors, and where $S^{l}=\left(S^{1}\right)^{(l)}$ for some simplicial model of $S^{1}$.In this paragraph we shall prove the following equivariant analogue of 1.16 and 1.17 .

Theorem 7.1. Let $X$ be a $\mathrm{C}_{r}$-space. The natural maps

$$
\begin{gathered}
\mathrm{Q}_{\mathrm{C}_{\mathrm{r}}}(|X|) \rightarrow \lim _{\rightarrow} \Omega^{l \mathscr{R}}\left(\left|\Gamma\left(S^{l \mathscr{R}} \wedge X\right)\right|\right) \\
|\Gamma(X)| \rightarrow \lim _{\rightarrow} \Omega^{l \mathscr{R}}\left(\left|\Gamma\left(S^{l \mathscr{R}} \wedge X\right)\right|\right)
\end{gathered}
$$

are $\mathrm{C}_{r}$-equivalences. In particular, $|\Gamma(X)| \simeq_{\mathrm{C}_{\mathrm{r}}} \mathrm{Q}_{\mathrm{C}_{\mathrm{r}}}(|X|)$.

For a cyclic space $Z, \Gamma(Z)$ is again a cyclic space, and $|Z|$ and $|\Gamma(Z)|$ have natural $S^{1}$-actions.

Corollary 7.2. For a cyclic space $Z,|\Gamma(Z)| \simeq_{\mathrm{C}_{\mathrm{r}}} \mathrm{Q}_{\mathrm{C}_{\mathrm{r}}}(|Z|)$.

Lemma 7.3. Let $s$ be a divisor in $r$. Then the inclusion

$$
\left(S^{l \mathscr{R}} \wedge X\right)^{\mathrm{C}_{s}} \rightarrow \operatorname{sd}_{r} \Gamma\left(S^{l \mathscr{R}} \wedge X\right)^{\mathrm{C}_{s}}
$$

is $2 l(r / s)-1$-connected.

Proof. By Lemma 3.14, it suffices to consider $\Gamma^{+}$instead of $\Gamma$. Let $Y=S^{l \mathscr{R}} \wedge X$ and filter the pair $\left(Y^{\mathrm{C}_{s}}, \operatorname{sd}_{r} \Gamma^{+}(Y)^{\mathrm{C}_{s}}\right)$ using the filtration 3.12. The filtration quotients are

$$
\begin{aligned}
& \operatorname{sd}_{r} \Gamma^{+}(n)(Y)^{\mathrm{C}_{s}} / \mathrm{sd}_{r} \Gamma^{+}(n-1)(Y)^{\mathrm{C}_{s}} \\
& \cong \bigvee_{\boldsymbol{a} \in \mathscr{A}(s, n)} \mathrm{E} \Sigma_{n}^{r / s}+\wedge_{\Sigma_{\Sigma_{n}}(\tilde{\boldsymbol{a}})} \bigwedge_{t \mid s}\left(Y^{\mathrm{C}_{t}}\right)^{\left(a_{t}\right)},
\end{aligned}
$$

cf. Lemma 3.6. Clearly $Y^{\mathrm{C}_{t}}$ is $l(r / t)$-connected, and therefore 7.4 is $\Sigma a_{t} l(r / t)-1=(r / s) \ln -1$ connected. The spectral sequence associated with the filtration shows that 


$$
\mathrm{H}_{i}\left(\operatorname{sd}_{r} \Gamma^{+}(Y)^{\mathrm{C}_{s}},(Y)^{\mathrm{C}_{s}}\right)=0, \quad \text { for } i \leq 2 l(r / s)-1,
$$

and the result now follows from the relative Hurewicz Theorem, as $\operatorname{sd}_{r} \Gamma^{+}(Y)^{\mathrm{C}_{s}}$ is simply connected for $l \geq 2$ by Theorem 3.1 .

The following lemma is [BHM, 3.11] with a misprint corrected.

Lemma 7.5. Let $f: X \rightarrow Y$ be a $\mathrm{C}_{r}$-map with $f^{\mathrm{C}_{s}} N(s)$-connected for each subgroup $\mathrm{C}_{s}$, and assume that

$$
N(s)-l(r / s) \leq N(t)-l(r / t)
$$

whenever $t$ divides $s$. Then the induced map

$$
f_{*}^{\mathrm{C}_{s}}: \operatorname{Map}_{\mathrm{C}_{s}}\left(S^{l \mathscr{R}}, X\right) \rightarrow \operatorname{Map}_{\mathrm{C}_{s}}\left(S^{l \mathscr{R}}, Y\right)
$$

is $N(s)-l(r / s)$ connected.

Proof of Theorem 7.1. We check that

$$
\mathrm{Q}_{\mathrm{C}_{\mathrm{r}}}(|X|)^{\mathrm{C}_{s}} \rightarrow \lim _{\rightarrow} \Omega^{l \mathscr{R}}\left(\left|\Gamma\left(S^{l \mathscr{R}} \wedge X\right)\right|\right)^{\mathrm{C}_{s}}
$$

is a non-equivariant equivalence for all subgroups $\mathrm{C}_{s}$. This follows from 7.3 and 7.5 , which show that

$$
\operatorname{Map}\left(S^{l \mathscr{R}}, S^{l \mathscr{R}} \wedge|X|\right)^{\mathrm{C}_{s}} \rightarrow \operatorname{Map}\left(S^{l \mathscr{R}},\left|\operatorname{sd}_{r} \Gamma\left(S^{l \mathscr{R}} \wedge X\right)\right|\right)^{\mathrm{C}_{s}}
$$

is $(l(r / s)-1)$-connected. This proves (i). To prove (ii), it again suffices to consider the induced map on fixed-points. We will prove by induction on $r$, that for any $\mathrm{C}_{r}$-space $X$ there is an equivalence

$$
\left|\operatorname{sd}_{r} \Gamma(X)^{\mathrm{C}_{r}}\right| \rightarrow \lim _{\rightarrow} \Omega^{l \mathscr{R}}\left(\left|\operatorname{sd}_{r} \Gamma\left(S^{l \mathscr{R}} \wedge X\right)\right|\right)^{\mathrm{C}_{r}} .
$$

For $r=1$ this is 1.17. The induction step goes through a series of reductions (1)-(4), and eventually reduces us to the case $X=\mathrm{C}_{r} / \mathrm{C}_{s+} \wedge S^{k}$ with $s \neq r$, which is taken care of by the Wirthmüller Isomorphism.

Step (1). The commutative diagram

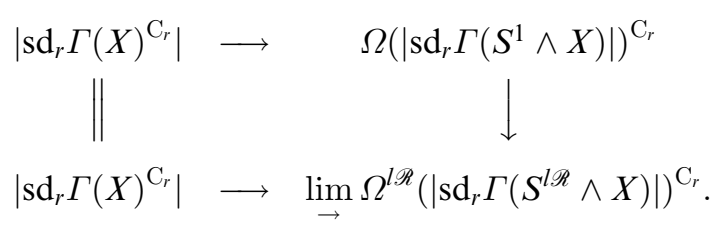

along with 4.4 reduces us to the case where $X$ is a suspension.

Step (2). We next reduce to the case where $X$ is $\mathrm{C}_{p}$-free for some prime $p$ dividing $r$. Let $\mathrm{C}_{q}$ be the Sylow- $p$ subgroup of $\mathrm{C}_{r}$, and consider the diagram 


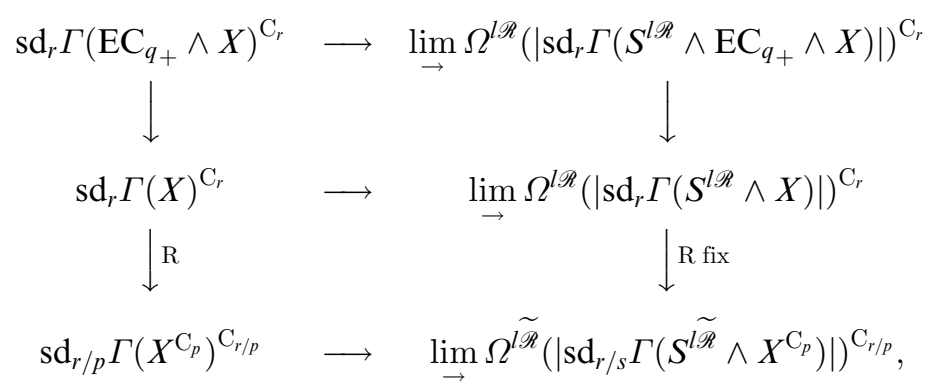

where $\widetilde{\mathscr{R}}$ is the regular representation for $\mathrm{C}_{r / s}$, and the fixed-point map fix was introduced in 0.1 .

The left column is a homotopy fibration by Corollary 5.5, and by anargument similar to that of [BHM, 5.17] this column would also be a homotopy fibration if $\Gamma$ was replaced by Q. Using (i) we conclude that the right vertical column is a homotopy fibration, and the five-lemma together with the induction hypothesis reduces us to the case where $X$ is $\mathrm{C}_{p}$-free.

Step (3). Since $X$ is the direct limit of its finite subcomplexes and the functors $\operatorname{sd}_{r} \Gamma$ and $\mathrm{Q}_{\mathrm{C}_{\mathrm{r}}}$ commute with such direct limits, we may further assume that $|X|$ has the form $Y \wedge S^{1}$, where $Y$ is a finite free based $\mathrm{C}_{r}$-CW complex.

Step (4). By (1), (2) and (3) it follows that $|X|$ has a finite filtration of topological spaces

$$
*=Y_{0} \subseteq Y_{1} \subseteq \ldots \subseteq Y_{n}=|X|,
$$

such that there are cofibration sequences $Y_{i-1} \rightarrow Y_{i} \rightarrow \mathrm{C}_{r} / \mathrm{C}_{s+} \wedge S^{k}$ with $k \geq 1$ and $s$ prime to $p$, cf. [A, 5.13]. We turn this into a cofibration-sequence of simplicial sets

$$
*=\sin Y_{0} \subseteq \sin Y_{1} \subseteq \ldots \subseteq \sin Y_{n}=\sin |X|,
$$

with homotopy cofibration sequences $\sin Y_{i-1} \rightarrow \sin Y_{i} \rightarrow \sin \left(\mathrm{C}_{r} / \mathrm{C}_{s+} \wedge S^{k}\right)$, and we get the diagram

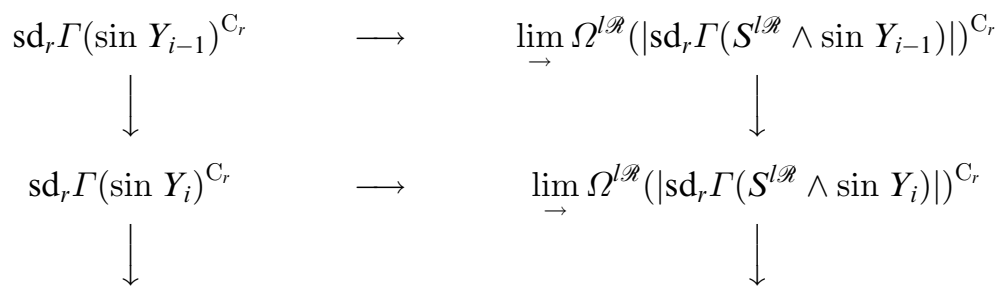

$$
\operatorname{sd}_{r} \Gamma\left(\sin \left(\mathrm{C}_{r} / \mathrm{C}_{s_{+}} \wedge S^{k}\right)\right)^{\mathrm{C}_{r}} \longrightarrow \lim \Omega^{l \mathscr{R}}\left(\left|\operatorname{sd}_{r} \Gamma\left(S^{l \mathscr{R}} \wedge \sin \left(\mathrm{C}_{r} / \mathrm{C}_{s_{+}} \wedge S^{k}\right)\right)\right|\right)^{\mathrm{C}_{r}}
$$

The columns are homotopy fibrations by 4.1 and (i). Using Lemma 2.9 and induction on $i$, we are reduced to the case $X=\mathrm{C}_{r} / \mathrm{C}_{s+} \wedge S^{k}, s \neq r$. However, 
this case follows immediately by the Wirthmüller Isomorphisms 6.2 and 6.3, together with (i) and the induction hypothesis:

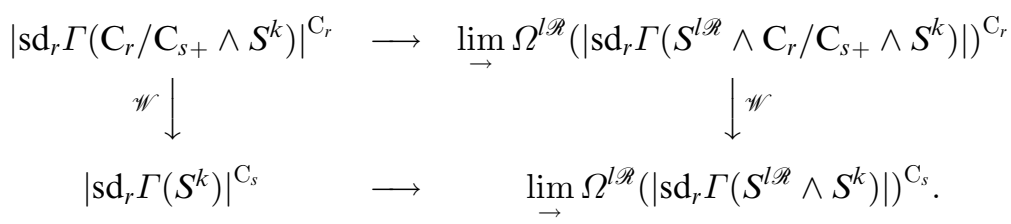

\section{REFERENCES}

[A] J. F. Adams, Prerequisites (on equivariant stable homotopy theory) for Carlsson's lecture, Algebraic Topology, Aarhus 1982, Lecture Notes in Math. 1051, 483-532.

[BE] M. Barratt and P. Eccles, $\Gamma^{+}$-structures-I: A free group functor for stable homotopy theory, Topology 13 (1974), 25-45.

[BHM] M. Bökstedt, W. C. Hsiang, and I. Madsen, The cyclotomic trace map and algebraic $K$ theory of spaces, Invent. Math. 111 (1993), 465-540.

[tD] T. tom Dieck, Orbittypen und äquivariante Homologie II, Arch. Math. 26 (1975), 650662.

[L] J. Loday, Cyclic Homology, Springer Verlag. 1992.

[May] J. P. May, Simplicial Objects in Algebraic Topology, Van Nostrand, New York, 1967.

[Sch1] C. Schlichtkrull, The transfer map in topological Hochschild homology, J. Pure Appl. Algebra 133 (1998), 289-316.

[Sch2] C. Schlichtkrull, On the induction map in topological cyclic homology, Preprint, Aarhus (1997).

[Sp] J. Spaliński, Strong homotopy theory of cyclic sets, J. Pure Appl. Algebra 99 (1995), 3552.

DEPARTMENT OF MATHEMATICS

AARHUS UNIVERSITY

DK-8000 AARHUS C

DENMARK

E-mail address: krull@mi.aau.dk 